\title{
CHRONIC HYPERTENSION IN PREGNANCY AND THE RISK OF CONGENITAL MALFORMATIONS: A COHORT STUDY
}

\author{
Brian T. Bateman, MD, MSc ${ }^{1,2}$, Krista F. Huybrechts, MS, $\mathrm{PhD}^{1}$, Michael A. Fischer, MD,

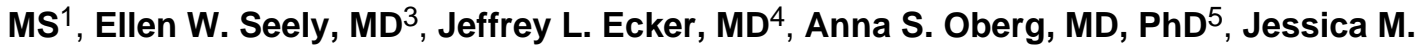 \\ Franklin, $\mathrm{PhD}^{1}$, Ms. Helen Mogun, $\mathbf{M S}^{1}$, and Sonia Hernandez-Diaz, MD, DrPH ${ }^{5}$ \\ ${ }^{1}$ Division of Pharmacoepidemiology and Pharmacoeconomics, Department of Medicine, Brigham \\ and Women's Hospital and Harvard Medical School, Boston, Massachusetts
}

\author{
${ }^{2}$ Department of Anesthesiology, Critical Care, and Pain Medicine, Massachusetts General \\ Hospital, Harvard Medical School, Boston, Massachusetts \\ ${ }^{3}$ Department of Medicine, Division of Endocrinology, Diabetes and Metabolism, Brigham and \\ Women's Hospital, Harvard Medical School, Boston, Massachusetts \\ ${ }^{4}$ Department of Obstetrics and Reproductive Sciences, Massachusetts General Hospital, Harvard \\ Medical School, Boston, Massachusetts \\ ${ }^{5}$ Department of Epidemiology, Harvard School of Public Health, Boston, Massachusetts
}

\begin{abstract}
Objective-Chronic hypertension is a common medical condition in pregnancy. The purpose of the study is to examine the association between maternal chronic hypertension and the risk of congenital malformations in the offspring.
\end{abstract}

Study Design-We defined a cohort of 878,126 completed pregnancies linked to infant medical records using the Medicaid Analytic Extract. The risk of congenital malformations was compared between normotensive controls and those with treated and untreated chronic hypertension. Confounding was addressed using propensity score matching.

Results-After matching, compared to normotensive controls, pregnancies complicated by treated chronic hypertension were at increased risk of congenital malformations (odds ratio (OR) $1.3,95 \%$ confidence interval (CI) 1.2 to 1.5 ), as were pregnancies with untreated chronic

(C) 2014 Elsevier Inc. All rights reserved.

CORRESPONDENCE TO: Brian T. Bateman, MD, MSc, Division of Pharmacoepidemiology \& Pharmacoeconomics, Department of Medicine, Brigham \& Women's Hospital, 1620 Tremont Street, Suite 3030, Boston, MA 02120, bbateman @ partners.org, Phone: 617-278-0930 | Fax: 617-232-8602.

SOURCE

Division of Pharmacoepidemiology \& Pharmacoeconomics

Department of Medicine

Brigham \& Women's Hospital

This is a PDF file of an unedited manuscript that has been accepted for publication. As a service to our customers we are providing this early version of the manuscript. The manuscript will undergo copyediting, typesetting, and review of the resulting proof before it is published in its final citable form. Please note that during the production process errors may be discovered which could affect the content, and all legal disclaimers that apply to the journal pertain.

FINANCIAL DISCLOSURES:

The other authors declare no potential conflict of interest. 
hypertension (OR 1.2, 95\% CI 1.1 to 1.3). In our analysis of organ-specific malformations, both treated and untreated chronic hypertension were associated with a significant increase in the risk of cardiac malformations (OR 1.6, 95\% CI 1.4 to 1.9 and OR 1.5, 95\% CI 1.3 to 1.7, respectively). These associations persisted across a range of sensitivity analyses.

Conclusion-There is a similar increase in the risk of congenital malformations (particularly cardiac malformations) associated with treated and untreated chronic hypertension that is independent of measured confounders. Studies evaluating the teratogenic potential of antihypertensive medications must control for confounding by indication. Fetuses and neonates of mothers with chronic hypertension should be carefully evaluated for potential malformations, particularly cardiac defects.

\section{Keywords}

hypertension; drug; epidemiology; pregnancy; birth defect

\section{Introduction}

Chronic hypertension is a common medical condition in pregnancy and its prevalence is rising as a larger number of parturients are obese and of advanced maternal age. ${ }^{1,2}$ As a consequence, exposure to antihypertensive medications during pregnancy, including in the first trimester when organogenesis occurs, is common and increasing. ${ }^{3-5}$

Certain classes of antihypertensive medications taken during the first trimester including beta blockers, diuretics, and angiotensin-converting enzyme (ACE) inhibitors have been associated with an increased risk of specific congenital malformations. ${ }^{6-11}$ However, several recent studies have suggested that it may be the underlying chronic hypertension that confers risk and not exposure to these medications per se, because: (1) an elevation in risk of malformations was observed across antihypertensive classes and/or (2) the association with certain medications was no longer present when medication users were compared to a control group that included untreated hypertensive patients. ${ }^{6,} 8,12$

Little is known about the role of chronic hypertension alone in conferring risk of congenital malformations and there has been a call from experts for further study in this area. ${ }^{13}$ Specifically, there are few data on whether chronic hypertension confers risk of malformations independent of other confounding factors (e.g., diabetes, maternal age, antihypertensive agents) and which specific malformations, if any, are associated with hypertension. Such information may be useful to clinicians in counseling patients and in guiding screening for malformations. It may also be important in informing the design of future studies of the teratogenic potential of antihypertensive medications. We therefore sought to examine the effect of chronic hypertension on the risk of congenital malformations in a large cohort of pregnancies in Medicaid beneficiaries. 


\section{Materials and Methods}

\section{Cohort}

The cohort was derived from the Medicaid Analytic eXtract (MAX), which contains information on Medicaid beneficiaries; Medicaid is the joint state and federal health insurance program for low-income individuals in the United States. MAX is a healthcare utilization database that records Medicaid enrollment and utilization claims, including those for inpatient admissions and outpatient visits, as well as outpatient pharmacy dispensing claims. Using the MAX data from 2000 to 2007, a cohort was created for the study of drug utilization and safety in pregnancy, as previously described in detail. ${ }^{14}$ The use of this database for research was approved by the Partners' Institutional Review Board (Boston, MA). Women with a claim indicating delivery were linked to infants within states using the Medicaid Case Number (which is generally shared by families) and the infant's date of birth. The woman's last menstrual period (LMP) was assigned using a validated algorithm based on diagnostic codes in the maternal and infant records. ${ }^{15}$

We restricted the cohort to women who were eligible for Medicaid continuously from 3 months prior to the estimated LMP month through one month postpartum. To ensure complete ascertainment of relevant claims, we restricted our analysis to women with $\geq 28$ days of enrollment each month, and without restricted benefits, private insurance, or certain state-specific managed care programs (that underreport claims to MAX). To allow for accurate capture of congenital malformations, we also required that the linked infants met the same Medicaid eligibility criteria as the mothers for at least 3 months following birth (unless they died in which case a shorter eligibility period was allowed). The source cohort included 891,699 completed pregnancies with linked infants.

We excluded women who were exposed to known teratogenic medications including lithium, antineoplastic agents, retinoids, or thalidomide from the estimated LMP through the date of delivery based on claims for dispensed medications or who had an infant with an inpatient or outpatient diagnosis code indicating the presence of a chromosomal abnormality. We also excluded women who were exposed to antihypertensive medications during the first trimester, but who lacked diagnosis codes indicating chronic hypertension due to significant risk of misclassifying the presence or absence of hypertension in these patients (as the women may have received the medications for other indications, for example beta blockers for migraine prophylaxis, or have hypertension that was not properly coded). The final analytic cohort included 878,126 pregnancies (Figure S1).

\section{Exposure}

Three groups of women were considered in the analysis: (1) women without chronic hypertension who did not receive antihypertensive medications in the first trimester, (2) women with chronic hypertension who were treated with an antihypertensive medication during the first trimester, and (3) women with chronic hypertension who were not treated with an antihypertensive medication during the first trimester.

Chronic hypertension was defined by codes recorded on two or more distinct dates indicating chronic or pre-existing hypertension recorded in the maternal inpatient or 
outpatient record anytime from 3 months prior to the LMP through delivery. The codes were derived from the International Classification of Disease $9^{\text {th }}$ edition-Clinical Modification codes and included codes 642.0x, 642.1x, 642.2x, 642.7x, and 401.xx through 405.xx. These codes are specific to chronic hypertension and are distinct from codes that indicate other hypertensive disorders of pregnancy including gestational hypertension and preeclampsia.

Antihypertensive exposure during the first trimester was defined by a filled prescription whose days supply overlapped the period from the LMP to 90 days after the LMP. In defining this exposure, we used prescriptions filled from 3 months prior to the LMP until 90 days post LMP. Duration of exposure was estimated based on the number of days supply. We accumulated days supply for consecutive prescriptions of the same medication if the medication was refilled prior to the day the prior prescription was expected to run out. The list of antihypertensive medications considered in the analysis can be found in Appendix A.

\section{Outcomes}

The primary outcome was defined as the presence of a major congenital malformation in the offspring. Major congenital malformations were defined based on having codes on two or more separate days in the infant inpatient or outpatient records during the first 3 months of life indicating an organ-specific class of malformations including central nervous system malformations, eye, ear, neck, and face malformations, cardiac malformations, respiratory malformations, cleft palate or lip, gastrointestinal malformations, genitourinary malformations, musculoskeletal malformations, or other malformations. We required two codes to define the presence of malformations to exclude cases in which a single mention may be recorded to justify a diagnostic test to rule out a condition. Our group has previously validated specific cardiac malformations using this approach with medical records; the positive predicted value was greater than $75 \% .{ }^{16}$ Secondary outcomes included each of the organ-specific malformations.

\section{Covariates}

We identified five groups of potential confounders in the cohort: maternal demographic characteristics, comorbid medical conditions, obstetric characteristics/conditions, maternal medications, and measures of healthcare utilization. Demographic characteristics assessed included age at delivery divided into six categories ( $\leq 9,20-24,25-29,30-34,35-39$, $\geq 40$ years), race/ethnicity (grouped as white (non-Hispanic), black (non-Hispanic), Hispanic, Asian/Pacific Islander, other, or missing), region of delivery (Northeast, South, West, or Midwest), and year of delivery.

Comorbid medical and obstetric conditions were identified by the presence of one or more diagnosis codes in the maternal inpatient or outpatient record from 3 months prior to the estimated LMP through delivery. These included pre-existing diabetes mellitus, chronic renal disease, obesity, tobacco use, alcohol abuse, and illicit drug use/abuse. Obstetric conditions included multiple gestations and gestational diabetes. Multiparity was defined based on the woman's Medicaid eligibility type; an eligibility classification of "adult with dependent children" indicated multiparity. 
Comorbidities and conditions were assessed until delivery because, while the relevant etiologic window for exposures leading to the development of congenital malformations is the first trimester, conditions present during the first trimester (chronic hypertension, diabetes, obesity, etc) may not always be coded with fidelity during this relatively brief window. Additionally, conditions that develop later in pregnancy (e.g., gestational diabetes) may be markers for risk factors that are present in the first trimester (maternal obesity or hyperglycemia). The validity of this approach rests on the assumption that the development of a congenital malformation in the infant does not lead to the development or preferential recording of these maternal conditions. While this is likely a safe assumption for the conditions assessed, we also conducted a sensitivity analysis to ensure that our findings were robust when the window during which exposure and covariates were ascertained was confined to the pre-pregnancy period and first trimester (see below).

Maternal medication exposures assessed included exposure to either insulin or oral hypoglycemic medications from 3 months prior to the LMP through delivery (which may be markers for the presence or severity of maternal pre-existing or gestational diabetes). We also identified and considered as a covariate in our analyses exposure to any potentially teratogenic medications during the first trimester including fluconazole, aminoglycosides, folic acid antagonists, methimazole, potassium iodide, tetracycline, danazol, misoprostol, statins, coumadin, and propylthiouracil which may act as confounders if women with chronic hypertension are exposed to these medications with a frequency that is different than normotensive women.

Finally, we considered measures of healthcare utilization during the 3 months prior to the LMP through the end of the first trimester which may be markers for overall health status, and thus have a relationship to the risk for birth defects. These included the number of distinct non-antihypertensive prescription and number of physician visits for any reason (both grouped as $0,1-3,>3$ ).

\section{Statistical analyses}

We determined the baseline characteristics of women without chronic hypertension who did not receive antihypertensive medications in the first trimester (controls), women with chronic hypertension who were exposed to an antihypertensive during the first trimester (treated chronic hypertensives), and women with chronic hypertension who were not treated with an antihypertensive during the first trimester (untreated chronic hypertensives) and summarized them as counts and proportions. We conducted two separate comparisons in our analyses: (1) controls versus treated chronic hypertensives and (2) controls versus untreated chronic hypertensives. In each analysis, we first determined the frequency of and unadjusted odds ratio and $95 \%$ confidence intervals (CIs) for the primary and secondary outcomes.

To account for the differences in baseline characteristics in the groups which are being compared, we performed propensity score analyses. In the first analysis (comparing controls and treated hypertensives), we used a logistic regression model to estimate the probability of being a treated hypertensive (as opposed to control) based on maternal demographic characteristics, comorbid medical conditions, obstetric characteristics/conditions, maternal medication exposures, and measures of healthcare utilization, as defined above, without 
further selection (this probability is the "propensity score"). Controls and treated chronic hypertensives were then matched on their propensity score in a fixed 3:1 ratio using a nearest neighbor algorithm with a maximum matching distance of 0.05 . The same approach was used for the comparison of controls with untreated chronic hypertensives. In these matched cohorts, we again estimated the odds ratio and 95\% CI for the primary and secondary outcomes.

\section{Sensitivity and Exploratory Analyses}

Because diabetes is such a strong risk factor for congenital malformations ${ }^{17,} 18$ and the possibility exists that there may be residual confounding even adjusting for the presence of diabetes (i.e., diabetes may be more severe in the hypertensive patients), we repeated our analysis excluding patients with any codes or medications indicating the presence of diabetes. Specifically, we excluded women who had any diagnosis codes indicating preexisting or gestational diabetes or who had prescriptions for insulin or oral diabetes medications from 3 months prior to the LMP through delivery. The total number of excluded patients was 88,937 . We then repeated the propensity-score matched analysis, focusing on the outcomes of overall congenital malformations and cardiac malformations (which were significantly associated with both treated and untreated hypertension in the primary analysis).

We performed a second sensitivity analysis excluding patients with preterm delivery. Preterm delivery has a complex relationship to congenital malformations as (1) infants with congenital malformations are more likely to deliver preterm, and (2) infants born preterm will sometimes have conditions that might be coded as malformations (e.g., patent ductus arterious, patent foramen ovale, undescended testes) that would have spontaneously resolved had the infant been carried to term. In the first scenario, controlling for prematurity could induce an association between hypertension or antihypertensives and malformations. In the second scenario, controlling for prematurity would be justified if we were interested in the direct effect of hypertension or antihypertensives not mediated through prematurity, assuming our analyses properly adjusted for shared risk factors of malformations and prematurity. ${ }^{19}$ In the primary analysis, we assumed the first scenario and did not adjust for preterm delivery. In this sensitivity analysis, we assume scenario two and condition on prematurity through restriction. The total number of pregnancies excluded in this analysis was 98,049 . We again repeated the main analysis for the outcomes of overall congenital malformations and cardiac malformations.

In the primary analysis, we collected information on covariates from prior to the LMP through delivery in order to improve our ascertainment of chronic hypertension and conditions that might confound the association of chronic hypertension and malformations. However, if a woman is diagnosed as carrying an infant with a malformation on prenatal ultrasound, it is possible that her medical conditions would be scrutinized more carefully and/or recorded more accurately in the medical record (surveillance bias). To ensure that this potential bias did not affect our results, we repeated our analysis defining chronic hypertension and the covariates used in the analysis based solely on codes recorded in the maternal record from 3 months prior to the LMP through the end of the first trimester. 
We required codes in the infant record recorded on separate days to define the presence of an organ-specific malformation in the primary analysis. This was done to minimize the risk of identifying malformations based on codes used to justify "rule-out" diagnostic tests. However, to test that our results were robust to this approach, we repeated the primary analysis with the outcome defined based on one or more code in infant inpatient or outpatient record from day of birth to day of life 90 .

To determine the potential effects of residual confounding in our analyses, we defined the strength (confounder-outcome relative risk) of a hypothetical residual confounder which, if present, would explain the effect of treated and untreated chronic hypertension on the risk of developing a congenital malformation. As the strength of confounders depends on the relative prevalence, we assumed a prevalence of 5\% among the normotensive controls and then a range of prevalences in the treated and untreated chronic hypertension patients (which were analyzed separately). ${ }^{20}$

As an exploratory analysis, we repeated the primary analysis, examining the association of treated and untreated hypertension and specific cardiac malformations including ventricular septal defect, right ventricular outflow obstruction, single ventricle, secundum atrial septal defect, conotruncal defect, and left ventricular outflow obstruction.

Analyses were performed in SAS 9.3 (SAS Institute, Cary, NC).

\section{Results}

Our primary cohort consisted of 878,126 completed pregnancies. Overall, 19,789 (2.3\%) had chronic hypertension; of these, 8307 (42.0\%) were treated with antihypertensive medication. As shown in Table 1, there were important baseline differences in patients without hypertension, with treated hypertension, and with untreated hypertension. Patients with chronic hypertension tended to be older, were more often African-American, and had a higher prevalence of preexisting diabetes, gestational diabetes, and renal disease. They were also more often exposed to insulin or other diabetic medications. These differences from normotensive controls were greater for patients with treated chronic hypertension than untreated hypertension. After propensity score matching, these imbalances were no longer present, with the absolute difference in the frequency of all covariates less than $2 \%$ (Table 2).

Congenital malformations in the offspring were observed in 29,934 (3.49\%) pregnancies without chronic hypertension or antihypertensive exposure, 491 (5.91\%) pregnancies with treated chronic hypertension, and 581 (5.06\%) pregnancies with untreated hypertension. The most commonly observed organ-specific malformations in the offspring were cardiac, followed by musculoskeletal, genitourinary, and gastrointestinal (Table S1). In the unadjusted analyses, compared to controls without chronic hypertension, those with treated chronic hypertension had a higher risk of any congenital malformation (OR 1.7, 95\% CI 1.6 to 1.9), as did those with untreated chronic hypertension (OR 1.5, 95\% CI 1.4 to 1.6). In the analysis of organ-specific malformations, those with treated hypertension had statistically significantly increased risk of infants affected by central nervous system malformations, 
cardiac malformations, respiratory malformations, genitourinary malformations, and other malformations (including malformations of the integument and malformations not otherwise specified) compared with controls. Women with untreated hypertension had a significantly higher risk of cardiac malformations and genitourinary malformations in their offspring compared to controls without chronic hypertension (Table 3). Point estimates for several other malformations were also increased in the treated and untreated hypertension groups, albeit with confidence intervals that intersected the null.

In the propensity score matched analysis, there was a higher risk of composite congenital malformations in the infants of both women with treated chronic hypertension (OR 1.3, 95\% CI 1.2 to 1.5 ) and untreated chronic hypertension (OR 1.2, 95\% CI 1.1 to 1.3) compared with normotensive controls. In the analysis of organ-specific malformations, in the propensity score matched analyses, both treated and untreated chronic hypertension were associated with increased risk for cardiac malformations (OR 1.6, 95\% CI 1.4 to 1.9 and OR $1.5,95 \%$ CI 1.3 to 1.7 , respectively) compared with normotensive controls. Point estimates for several other malformations, including central nervous system malformations and respiratory malformations, were increased for women with both treated and untreated chronic hypertension, although confidence intervals for these associations intersected the null (Table 3).

Across each of the sensitivity analyses, both treated and untreated chronic hypertension were associated with increased risk of the composite congenital malformation and cardiac malformation endpoints in the offspring, although for some of these associations the confidence intervals intersected the null (Table 4). The estimate for the association of untreated chronic hypertension and composite congenital malformations and cardiac malformations was somewhat attenuated when preterm deliveries were excluded.

We defined the strength of a hypothetical residual confounder that could have produced the observed effect of treated chronic hypertension on the risk of overall malformations from the primary analysis (adjusted OR 1.3) and then repeated this analysis for untreated hypertension (adjusted OR 1.2). For both analyses we assumed a prevalence of the hypothetical confounder of 5\% in the normotensive control group. A confounder-outcome risk ratio of 9.6 would be required to produce the observed effect of treated chronic hypertension if the prevalence of the confounder in the treated hypertension group was $10 \%$, a risk ratio of 3.2 if the prevalence was $20 \%$, and a risk ratio of 2.3 if the prevalence was $30 \%$. For the untreated chronic hypertension effect to be explained by an unmeasured confounder, a confounder-outcome risk ratio of 6.6 would be required if the prevalence of the confounder in the untreated hypertension group was $10 \%$, a risk ratio of 2.6 if the prevalence was $20 \%$, and a risk ratio of 1.9 if the prevalence was 30\% (see Figure S2).

Having demonstrated an association between cardiac malformations and both treated and untreated hypertension, we explored the association with specific types of cardiac malformations. Point estimates for most malformations examined were modestly increased for both treated and untreated chronic hypertensive patients, although in the setting of small numbers most confidence intervals were wide and intersected the null (Table 4). 


\section{Comment}

In this cohort of 878,126 pregnancies, we found that both treated and untreated maternal chronic hypertension were associated with a similar 20 to $30 \%$ increase in the risk of congenital malformations in the infant after adjusting for confounding factors as compared with normotensive controls. This increase in risk persisted across a number of sensitivity analyses. In our evaluation of organ-specific malformations, both treated and untreated chronic hypertension were associated with an increased risk of cardiac malformations.

Chronic hypertension has not been traditionally identified as a risk factor for congenital malformations in the offspring. As such, most prior studies of the teratogenic potential of antihypertensive medications have not carefully accounted for potential confounding by indication. Our findings therefore have important implications in informing the way in which studies of antihypertensive drug safety in pregnancy should be performed going forward. They also identify congenital malformations as an important complication associated with chronic hypertension, which may have clinical implications for antenatal screening in affected women.

There are few studies that have explored the role of hypertension in conferring risk of congenital malformations in the offspring. A cohort study by Li et al. of the teratogenic potential of angiotensin converting enzyme inhibitors in the first trimester found that a comparison group of patients with untreated hypertension, as defined by diagnosis codes indicating hypertension from prior to pregnancy through delivery, had an increased risk of congenital heart defects and neural tube defects. ${ }^{12}$ Caton et al., in a case-control study, found that untreated hypertension (again, defined by hypertension anytime during pregnancy) was associated with an increased point estimate for cardiovascular malformations, but with wide confidence intervals that intersected the null. ${ }^{6}$

Our findings confirm and extend these observations from previous studies. First, we examine the role specifically of chronic hypertension in conferring risk of malformations whereas prior studies examined all pregnancy-related hypertensive disorders together. ${ }^{6,12}$ Chronic hypertension is the most etiologically relevant type of hypertension to examine because, in contrast to other hypertensive disorders of pregnancy such as gestational hypertension or preeclampsia, it is present in the first trimester (the period during which organogenesis occurs). Second, our study examines a full range of organ-specific congenital malformations in relation to hypertension, finding that chronic hypertension is associated most strongly with cardiac malformations. Third, our study carefully controls for a wide range of potential confounders of the association between chronic hypertension and congenital malformations including comorbid conditions, as well as other relevant medication exposures and markers of overall health status and healthcare utilization. Finally, the large size of our cohort allows us to make relatively precise estimates regarding the risk of malformations associated with treated and untreated hypertension.

The association between chronic hypertension and malformations may be biologically plausible. Chronic hypertension can result in uteroplacental insufficiency which accounts, at least in part, for its association with intrauterine growth restriction, intrauterine fetal demise 
and superimposed preeclampsia. ${ }^{2,21-25}$ Uteroplacental insufficiency can lead to compromised blood flow to the developing fetus which if present in early pregnancy may, in turn, increase the risk for certain types of malformations, particularly cardiovascular malformations ${ }^{6,26}$ previous authors have postulated that this mechanism may explain the link between hypertension and cardiac malformations. ${ }^{6}$ That said, much remains to be learned about the biological mechanisms that explain the observed association between chronic hypertension and malformations and whether controlling hypertension would reduce the risk. Our results, coupled with the rapidly rising prevalence of chronic hypertension in pregnancy, motivate such work. ${ }^{1,2}$

Our findings also significantly inform the interpretation of prior studies examining the teratogenic potential of antihypertensive medications. ${ }^{6-11}$ Studies that identify an association of these medications with malformations but which do not control for potential confounding by indication must be interpreted with caution. Future studies examining the role of specific antihypertensive medications will need to carefully choose a comparator group of patients with the same underlying condition in order to yield valid results.

The results of the study may inform the performance of antenatal screening tests. Guideline groups have advocated for the use of fetal echocardiography based on maternal comorbidities that confer increased risk of congenital cardiac malformations. ${ }^{27}$ The crude risks observed in our study suggest that women with chronic hypertension have a 50 to $70 \%$ greater risk of cardiac malformations compared to normotensive controls (which attenuated to 20 to $30 \%$ after controlling for confounders), suggesting that this group may benefit from such testing. Future work will need to establish the clinical benefits and cost-effectiveness of performing fetal echocardiography in this population.

Our results should be interpreted in the context of limitations inherent in its design. Although the MAX database contains detailed information on patient demographics, outpatient medication utilization, and inpatient and outpatient diagnoses, it is healthcare utilization data that is not collected primarily for research purposes and thus lacks some relevant clinical details. For example, the patients' blood pressures are not directly recorded in the MAX and thus we are reliant on physicians' diagnosis of chronic hypertension in defining exposure. Likewise, the database does not contain detailed information describing the malformations diagnosed in the infants and the definition of malformations is confined to those identified in early infancy. To overcome these potential limitations, we defined the presence of both the chronic hypertension and the outcome of malformations based on two instances of coding in the maternal or infant record, respectively, meaning that a physician diagnosed these conditions on two separate occasions. Such an approach is expected to define both exposure and outcome with high specificity. When the outcome is defined with high specificity (even in the presence of limited sensitivity), results from an observational study will yield unbiased estimates of relative risk of the association of the exposure with the outcome. ${ }^{28}$ Further, any misclassification of the outcome in the study would be expected to be non-differential in the groups compared. As a consequence, if misclassification of outcome is present in our study, estimates of the association between chronic hypertension and malformations would, if anything, be biased to the null. 
In studying the association between a chronic maternal condition and malformations, there is the possibility that the findings may be subject to ascertainment bias if the infants of women with the condition are examined for malformations more carefully than those of unaffected women; however, this bias is unlikely to be playing a major role in the findings of the present study since chronic hypertension has not traditionally been thought in the malformation to be a risk factor for malformations, as well as the fact that many of the specific cardiac malformations associated with hypertension (see Table 4) present a threat to the life of the infant and/or require surgical intervention. The timing of the last menstrual period is not recorded directly in the database, but has to be estimated using an algorithm based on diagnostic codes. While the algorithm has been demonstrated to perform well in claims data, misclassification of the last menstrual period in some patients is possible. This would not affect the identification of patients with chronic hypertension, but it may lead to some small degree of misclassification of patients' treatment status. Additionally, while we follow infants for 90 days after birth when the majority of major malformations are likely to be detected, it is possible that some defects will only be detected later in infancy, leading us to underestimate the prevalence of malformations in the cohort. Further, our study was conducted in a population of low income Medicaid beneficiaries. While the observed effect of chronic hypertension on the risk of malformations is very likely to be generalizable to other populations, even if this were not the case, the results would be of interest given the large proportion of births in the US covered by Medicaid. Finally, we use propensity score matching to control for confounders in our analysis. The estimates of the effect of treated and untreated hypertension on the risks of malformation should be interpreted as generalizable to populations with characteristics of the matched populations, as shown in Table 2.

While we were careful to identify potential confounders of the association between chronic hypertension and malformations using maternal inpatient and outpatient diagnostic claims, medications, and healthcare utilization variables, it remains possible that residual bias is present due to unmeasured or unknown confounders. Our analysis of the potential effect of a hypothetical confounder suggests that for an unobserved confounder to explain our results, it would need to have a strong association with the outcome and be highly prevalent in the exposed group. While the presence of a single highly prevalent unmeasured confounder of strong effect is unlikely given our longitudinal assessment of all women in the cohort from 3 months prior to the LMP through delivery, it cannot be excluded that several unmeasured or not perfectly measured confounders might together significantly attenuate the observed associations. For example, while we accounted for confounding by diabetes (both gestational and pre-existing) through the use of both diagnosis codes and treatment with insulin or oral diabetes medications, impaired glucose tolerance which does not meet the threshold for the diagnosis of pre-existing or gestational diabetes may be more common in women with chronic hypertension and thus represent a source of residual confounding. Our datasource also has imperfect data on overweight and obesity, which may represent another source of potential residual confounding. Future work using other data sources will thus be needed to confirm these results. That said, the implications of our results for the design of drug safety studies in pregnancy (need to control for confounding by indication, as the confounders measured in our dataset are typical of those available in datasets used to study 
drug safety) and for clinical practice (need for close screening for malformation in women with hypertension) remain whether or not the observed association is strictly causal.

Our study also cannot directly address whether treatment of chronic hypertension in pregnancy will decrease the risk of malformations. However, if the mechanism underlying the association of chronic hypertension and malformations is, in fact, uteroplacental insufficiency, it is unlikely that treatment will be effective in doing so. The risks of preeclampsia and small for gestational age associated with chronic hypertension, which may also be secondary to uteroplacental insufficiency, are not decreased through the use of antihypertensive therapy. ${ }^{29}$ Likewise, while the estimates of risk of malformations was similar for both treated and untreated chronic hypertension, our study cannot exclude the possibility that certain antihypertensives are teratogenic. Future studies will need to focus on evaluating the safety of particular antihypertensives while carefully accounting for the confounders that are important to those agents.

Our findings suggest that chronic hypertension, whether treated or untreated, has an independent association with the development of major congenital malformations (particularly cardiac malformations). As in all observational studies, we cannot exclude the possibility of residual confounding and additional studies replicating these findings are necessary before chronic hypertension should be considered an established risk factor for congenital malformations. Future research is also needed to define the biological basis for this association and to determine how it might be modified. Studies of the teratogenic potential of antihypertensive medications in pregnancy need to be attentive to confounding by indication. Clinically, the findings suggest that hypertensive women may benefit from more intensive screening for cardiac malformations in the fetus/neonate.

\section{Supplementary Material}

Refer to Web version on PubMed Central for supplementary material.

\section{ACKNOWLEDGEMENTS}

Thanks to Cora Allen-Coleman for her work as a research assistant on this project.

Research reported in this publication was supported by the Eunice Kennedy Shriver National Institute of Child Health \& Human Development of the NIH (Bethesda, Maryland, United States) under Award Number K08HD075831 (BTB). MAX pregnancy cohort was supported by the Agency for Healthcare Research and Quality (AHRQ) (Grant R01HS018533). The content is solely the responsibility of the authors and does not necessarily represent the official views of the National Institutes of Health.

SHD has consulted for Novartis, GlaxoSmithKline-Biologics (Middlesex, England, United Kingdom) and AstraZeneca (London, England, United Kingdom) for unrelated projects.

\section{References}

1. Kuklina EV, Ayala C, Callaghan WM. Hypertensive disorders and severe obstetric morbidity in the united states. Obstet Gynecol. 2009; 113:1299-1306. [PubMed: 19461426]

2. Bateman BT, Bansil P, Hernandez-Diaz S, Mhyre JM, Callaghan WM, Kuklina EV. Prevalence, trends, and outcomes of chronic hypertension: A nationwide sample of delivery admissions. Am J Obstet Gynecol. 2012; 206:134. e131-138. [PubMed: 22177190] 
3. Andrade SE, Raebel MA, Brown J, Lane K, Livingston J, Boudreau D, Rolnick SJ, Roblin D, Smith DH, Dal Pan GJ, Scott PE, Platt R. Outpatient use of cardiovascular drugs during pregnancy. Pharmacoepidemiol. Drug Saf. 2008; 17:240-247. [PubMed: 18200619]

4. Bateman BT, Hernandez-Diaz S, Huybrechts KF, Palmsten K, Mogun H, Ecker JL, Fischer MA. Patterns of outpatient antihypertensive medication use during pregnancy in a medicaid population. Hypertension. 2012; 60:913-920. [PubMed: 22966012]

5. Xie RH, Guo Y, Krewski D, Mattison D, Nerenberg K, Walker MC, Wen SW. Trends in using betablockers and methyldopa for hypertensive disorders during pregnancy in a canadian population. Eur J Obstet Gynecol Reprod Biol. 2013; 171:281-285. [PubMed: 24139131]

6. Caton AR, Bell EM, Druschel CM, Werler MM, Lin AE, Browne ML, McNutt LA, Romitti PA, Mitchell AA, Olney RS, Correa A. Antihypertensive medication use during pregnancy and the risk of cardiovascular malformations. Hypertension. 2009; 54:63-70. [PubMed: 19433779]

7. Yakoob MY, Bateman BT, Ho E, Hernandez-Diaz S, Franklin JM, Goodman JE, Hoban RA. The risk of congenital malformations associated with exposure to beta-blockers early in pregnancy: A meta-analysis. Hypertension. 2013; 62:375-381. [PubMed: 23753416]

8. Lennestal R, Otterblad Olausson P, Kallen B. Maternal use of antihypertensive drugs in early pregnancy and delivery outcome, notably the presence of congenital heart defects in the infants. Eur J Clin Pharmacol. 2009; 65:615-625. [PubMed: 19198819]

9. Puho EH, Szunyogh M, Metneki J, Czeizel AE. Drug treatment during pregnancy and isolated orofacial clefts in hungary. Cleft Palate Craniofac J. 2007; 44:194-202. [PubMed: 17328645]

10. Kallen BA, Otterblad Olausson P. Maternal drug use in early pregnancy and infant cardiovascular defect. Reprod Toxicol. 2003; 17:255-261. [PubMed: 12759093]

11. Cooper W, Hernandez-Diaz S, Arbogast P, Dudley J, Dyer S, Gideon P, Hall K, Ray W. Major congenital malformations after first-trimester exposure to ace inhibitors. New England Journal of Medicine. 2006; 354:2443-2451. [PubMed: 16760444]

12. Li DK, Yang C, Andrade S, Tavares V, Ferber JR. Maternal exposure to angiotensin converting enzyme inhibitors in the first trimester and risk of malformations in offspring: A retrospective cohort study. BMJ. 2011; 343:d5931. [PubMed: 22010128]

13. Mitchell AA. Fetal risk from ace inhibitors in the first trimester. BMJ. 2011; 343:d6667. [PubMed: 22010131]

14. Palmsten K, Huybrechts KF, Mogun H, Kowal MK, Williams PL, Michels KB, Setoguchi S, Hernandez-Diaz S. Harnessing the medicaid analytic extract (max) to evaluate medications in pregnancy: Design considerations. PLoS One. 2013; 8:e67405. [PubMed: 23840692]

15. Margulis AV, Setoguchi S, Mittleman MA, Glynn RJ, Dormuth CR, Hernandez-Diaz S. Algorithms to estimate the beginning of pregnancy in administrative databases. Pharmacoepidemiol Drug Saf. 2013; 22:16-24. [PubMed: 22550030]

16. Palmsten K, Huybrechts KF, Kowal MK, Mogun H, Hernández-Díaz S. Validity of maternal and infant outcomes within nationwide medicaid data. Pharmacoepidemiol Drug Saf. In press.

17. Casson IF, Clarke CA, Howard CV, McKendrick O, Pennycook S, Pharoah PO, Platt MJ, Stanisstreet M, van Velszen D, Walkinshaw S. Outcomes of pregnancy in insulin dependent diabetic women: Results of a five year population cohort study. BMJ. 1997; 315:275-278. [PubMed: 9274545]

18. Allen VM, Armson BA, Wilson RD, Allen VM, Blight C, Gagnon A, Johnson JA, Langlois S, Summers A, Wyatt P, Farine D, Armson BA, Crane J, Delisle MF, Keenan-Lindsay L, Morin V, Schneider CE, Van Aerde J, Society of O, Gynecologists of C. Teratogenicity associated with preexisting and gestational diabetes. J Obstet Gynaecol Can. 2007; 29:927-944. [PubMed: 17977497]

19. Hernandez-Diaz S, Schisterman EF, Hernan MA. The birth weight "paradox" uncovered? Am J Epidemiol. 2006; 164:1115-1120. [PubMed: 16931543]

20. Schneeweiss S. Sensitivity analysis and external adjustment for unmeasured confounders in epidemiologic database studies of therapeutics. Pharmacoepidemiol Drug Saf. 2006; 15:291-303. [PubMed: 16447304]

21. Rey E, Couturier A. The prognosis of pregnancy in women with chronic hypertension. Am J Obstet Gynecol. 1994; 171:410-416. [PubMed: 8059820] 
22. Sibai BM, Lindheimer M, Hauth J, Caritis S, VanDorsten P, Klebanoff M, MacPherson C, Landon M, Miodovnik M, Paul R, Meis P, Dombrowski M. Risk factors for preeclampsia, abruptio placentae, and adverse neonatal outcomes among women with chronic hypertension. National institute of child health and human development network of maternal-fetal medicine units. N Engl J Med. 1998; 339:667-671. [PubMed: 9725924]

23. Allen VM, Joseph K, Murphy KE, Magee LA, Ohlsson A. The effect of hypertensive disorders in pregnancy on small for gestational age and stillbirth: A population based study. BMC Pregnancy Childbirth. 2004; 4:17. [PubMed: 15298717]

24. Sibai BM. Chronic hypertension in pregnancy. Obstetrics and Gynecology. 2002; 100:369-377. [PubMed: 12151166]

25. Bateman BT, Simpson LL. Higher rate of stillbirth at the extremes of reproductive age: A large nationwide sample of deliveries in the united states. Am J Obstet Gynecol. 2006; 194:840-845. [PubMed: 16522422]

26. Clark EB. Pathogenetic mechanisms of congenital cardiovascular malformations revisited. Semin Perinatol. 1996; 20:465-472. [PubMed: 9090774]

27. Rychik J, Ayres N, Cuneo B, Gotteiner N, Hornberger L, Spevak PJ, Van Der Veld M. American society of echocardiography guidelines and standards for performance of the fetal echocardiogram. Journal of the American Society of Echocardiography : official publication of the American Society of Echocardiography. 2004; 17:803-810. [PubMed: 15220910]

28. Schneeweiss S, Avorn J. A review of uses of health care utilization databases for epidemiologic research on therapeutics. Journal of Clinical Epidemiology. 2005; 58:323-337. [PubMed: 15862718]

29. Abalos E, Duley L, Steyn DW, Henderson-Smart DJ. Antihypertensive drug therapy for mild to moderate hypertension during pregnancy. Cochrane Database Syst Rev. 2007 CD002252.

\section{Appendix A: Antihypertensive medications included in the analysis}

1. Diuretics

a. Thiazides

Bendroflumethiazide

Benzthiazide

Chlorothiazide

Chlorothiazide Sodium

Chlorthalidone

Cyclothiazide

Hydrochlorothiazide

Hydroflumethiazide

Indapamide

Methyclothiazide

Metolazone

Polythiazide

Quinethazone

Trichlormethiazide 
b. Potassium-Sparing Agents

Amiloride Hydrochloride

Spironolactone

Triamterene

c. Acetazolamide

Acetazolamide

2. Adrenergic Inhibitors

a. Peripheral Agents

Guanadrel Sulfate

Guanethidine Sulfate

Reserpine

b. Central Alpha-Antagonists

Clonidine Hydrochloride

Guanabenz Acetate

Guanfacine Hydrochloride

Methyldopa

Methyldopate Hydrochloride

Phenoxybenzamine Hydrochloride

Phentolamine Hydrochloride

Tolazoline Hydrochloride

c. Alpha Blockers

Doxazosin Mesylate

Prazosin Hydrochloride

Terazosin Hydrochloride

d. Beta Blockers

Acebutolol Hydrochloride

Atenolol

Betaxolol Hydrochloride

Bisoprolol Fumarate

Carteolol Hydrochloride

Esmolol Hydrochloride

Metoprolol Succinate 

Metoprolol Tartrate
Nadolol
Penbutolol Sulfate
Pindolol
Propranolol Hydrochloride
Sotalol Hydrochloride
Timolol Maleate
e. Combined Alpha And Beta Blockers
Carvedilol
Labetalol Hydrochloride

3. Direct Vasodilators

Hydralazine Hydrochloride

Minoxidil

4. Calcium Channel Antagonists

a. Nondihydropyridines

Diltiazem Hydrochloride

Diltiazem Malate

Mibefradil Di-Hydrochloride

Verapamil Hydrochloride

b. Dihydropyridines

Amlodipine Besylate

Bepridil Hydrochloride

Felodipine

Isradipine

Nicardipine Hydrochloride

Nifedipine

Nimodipine

Nisoldipine

5. ACE Inhibitors

Benazepril Hydrochloride

Captopril

Enalapril Maleate

Am J Obstet Gynecol. Author manuscript; available in PMC 2016 March 01. 
Enalaprilat

Enalaprilat Dihydrate

Fosinopril Sodium

Lisinopril

Moexipril Hydrochloride

Perindopril Erbumine

Quinapril Hydrochloride

Ramipril

Trandolapril

6. Angiotensin II Receptor Blockers

Candesartan Cilexetil

Eprosartan Mesylate

Irbesartan

Losartan Potassium

Olmesartan Medoxomil

Telmisartan

Valsartan

7. Combination Drugs

a. Beta Blockers And Diuretics

Bendroflumethiazide/Nadolol

Chlorthalidone/Atenolol

Hydrochlorothiazide/Bisoprolol Fumarate

Hydrochlorothiazide/Labetalol Hydrochloride

Hydrochlorothiazide/Metoprolol Tartrate

Hydrochlorothiazide/Propranolol

Hydrochlorothiazide/Propranolol Hydrochloride

Hydrochlorothiazide/Timolol

b. ACE Inhibitors And Diuretics

Benazepril Hydrochloride/Hydrochlorothiazide

Captopril/Hydrochlorothiazide

Enalapril Maleate/Hydrochlorothiazide

Fosinopril Sodium/Hydrochlorothiazide 
Lisinopril/Hydrochlorothiazide

Moexipril Hydrochloride/Hydrochlorothiazide

Quinapril Hydrochloride/Hydrochlorothiazide

c. Angiotensin II Receptor Antagonists And Diuretics

Candesartan Cilexetil/Hydrochlorothiazide

Irbesartan/Hydrochlorothiazide

Losartan Potassium/Hydrochlorothiazide

Telmisartan/Hydrochlorothiazide

Valsartan/Hydrochlorothiazide

d. Calcium Antagonists And ACE Inhibitors

Benazepril Hydrochloride/Amlodipine Besylate

Enalapril Maleate/Diltazem Maleate

Enalapril Maleate/Felodipine

Trandolapril/Verapamil Hydrochloride

e. Other Combinations

Bendroflumethiazide/Potassium Chloride

Cryptenamine/Methylclothiazide

Hydrochlorothiazide/Spironolactone

Spironolactone/Hydrochlorothiazide

Hydrochlorothiazide/Triamterene

Hydrochlorothiazide/Amiloride Hydrochloride

Clonidine Hydrochloride/Chlorthalidone

Deserpidine/Hydrochlorothiazide

Deserpidine/Methyclothiazide

Guanethidine Sulfate/Hydrochlorothiazide

Methyldopa/Chlorothiazide

Methyldopa/Hydrochlorothiazide

Reserpine/Benzthiazide

Reserpine/Chlorothiazide

Reserpine/Chlorthalidone

Reserpine/Hydrochlorothiazide

Reserpine/Hydroflumethiazide 
Reserpine/Methyclothiazide

Reserpine/Polythiazide

Reserpine/Quinethazone

Reserpine/Trichlormethiazide

Hydralazine Hydrochloride/Hydrochlorothiazide

Hydralazine Hydrochloride/Reserpine

Hydralazine Hydrochloride/Reserpine/Hydrochlorothiazide

Hydralaz/Reserpine/Hydrochlorothiazide

Hydralazine Hydrochloride/Hydrochlorothiazide

Prazosin Hydrochloride/Polythiazide

Methylclothiazide/Pargyline

Rauwolfia Serpentina/Bendroflumethiazide

Rauwolfia/Bendroflumethiazide/Potassium

8. Miscellaneous

Diazoxide

Metyrosine

Reserpine/Mannitol Hexanitrate 


\section{Table 1}

Baseline characteristics of study patients stratified as (1) without chronic hypertension, (2) with chronic hypertension and $1^{\text {st }}$ trimester antihypertensive exposure, and (3) with chronic hypertension and without $1^{\text {st }}$ trimester antihypertensive exposure. Values are numbers (percentages).

\begin{tabular}{|c|c|c|c|}
\hline & $\begin{array}{c}\text { Without } \\
\text { chronic } \\
\text { hypertension }\end{array}$ & $\begin{array}{l}\text { Treated chronic } \\
\text { hypertensives }\end{array}$ & $\begin{array}{l}\text { Untreated chronic } \\
\text { hypertensives }\end{array}$ \\
\hline Total & 858,337 & 8,307 & 11,482 \\
\hline \multicolumn{4}{|l|}{ Age group, years } \\
\hline$\leq 19$ & 256973 (29.9) & $402(4.8)$ & $2063(18)$ \\
\hline $20-24$ & $308140(35.9)$ & $1539(18.5)$ & 3435 (29.9) \\
\hline $25-29$ & $173291(20.2)$ & $2396(28.8)$ & $2958(25.8)$ \\
\hline $30-34$ & $78721(9.2)$ & $2201(26.5)$ & 1794 (15.6) \\
\hline $35-39$ & 33987 (4) & $1361(16.4)$ & $949(8.3)$ \\
\hline$\geq 40$ & $7225(0.8)$ & $408(4.9)$ & $283(2.5)$ \\
\hline \multicolumn{4}{|l|}{ Race/ethnicity } \\
\hline White, Non-Hispanic & 349369 (40.7) & $2684(32.3)$ & 4313 (37.6) \\
\hline Black, Non-Hispanic & $289867(33.8)$ & $4337(52.2)$ & $5179(45.1)$ \\
\hline Hispanic & $131057(15.3)$ & $689(8.3)$ & $1110(9.7)$ \\
\hline Asian & $29681(3.5)$ & $185(2.2)$ & $257(2.2)$ \\
\hline Other & $41039(4.8)$ & $248(3)$ & $402(3.5)$ \\
\hline Unknown & $17324(2.0)$ & $164(2.0)$ & $221(1.9)$ \\
\hline \multicolumn{4}{|l|}{ Patient characteristics } \\
\hline Pre-existing $\mathrm{DM}^{\dagger}$ & $33694(3.9)$ & $1989(23.9)$ & $1669(14.5)$ \\
\hline Gestational $\mathrm{DM}^{\dagger}$ & $67081(7.8)$ & $2122(25.5)$ & $2216(19.3)$ \\
\hline Chronic renal disease & $9362(1.1)$ & $404(4.9)$ & $517(4.5)$ \\
\hline Tobacco use & $71216(8.3)$ & $661(8.0)$ & $1007(8.8)$ \\
\hline \multicolumn{4}{|l|}{ Medication exposure } \\
\hline Insulin & $15648(1.8)$ & $1343(16.2)$ & $1005(8.8)$ \\
\hline Oral diabetes medications & $16682(1.9)$ & $2042(24.6)$ & $1010(8.8)$ \\
\hline
\end{tabular}


Table 2

Baseline characteristics of study patients after propensity score matching. Values are numbers (percentages).

\begin{tabular}{|c|c|c|c|c|}
\hline & \multicolumn{2}{|c|}{ Comparison 1} & \multicolumn{2}{|c|}{ Comparison 2} \\
\hline & $\begin{array}{l}\text { Without chronic } \\
\text { hypertension }\end{array}$ & $\begin{array}{l}\text { Treated chronic } \\
\text { hypertensives }\end{array}$ & $\begin{array}{l}\text { Without chronic } \\
\text { hypertension }\end{array}$ & $\begin{array}{l}\text { Untreated chronic } \\
\text { hypertensives }\end{array}$ \\
\hline Total & 23,427 & 7,809 & 34,434 & 11,478 \\
\hline \multicolumn{5}{|l|}{ Age group, years } \\
\hline$\unlhd 9$ & $1082(4.6)$ & $402(5.2)$ & 6069 (17.6) & $2063(18)$ \\
\hline $20-24$ & 4590 (19.6) & $1532(19.6)$ & 10185 (29.6) & 3434 (29.9) \\
\hline $25-29$ & $6961(29.7)$ & $2322(29.7)$ & $9084(26.4)$ & $2957(25.8)$ \\
\hline $30-34$ & $6178(26.4)$ & $2006(25.7)$ & $5369(15.6)$ & $1793(15.6)$ \\
\hline $35-39$ & $3563(15.2)$ & $1187(15.2)$ & $2899(8.4)$ & $948(8.3)$ \\
\hline$\geq 40$ & $1053(4.5)$ & $360(4.6)$ & $828(2.4)$ & $283(2.5)$ \\
\hline \multicolumn{5}{|l|}{ Race/ethnicity } \\
\hline White, Non-Hispanic & $7989(34.1)$ & $2585(33.1)$ & $13362(38.8)$ & $4313(37.6)$ \\
\hline Black, Non-Hispanic & $11793(50.3)$ & $3967(50.8)$ & $15425(44.8)$ & $5175(45.1)$ \\
\hline Hispanic & $2025(8.6)$ & $677(8.7)$ & $3205(9.3)$ & $1110(9.7)$ \\
\hline Asian & $481(2.1)$ & $185(2.4)$ & $639(1.9)$ & $257(2.2)$ \\
\hline Other & $692(3)$ & $241(3.1)$ & $1157(3.4)$ & $402(3.5)$ \\
\hline Unknown & 447 (1.9) & $154(2)$ & $646(1.9)$ & $221(1.9)$ \\
\hline \multicolumn{5}{|l|}{$\begin{array}{l}\text { Patient } \\
\text { characteristics }\end{array}$} \\
\hline Pre-existing $\mathrm{DM}^{\dagger}$ & 4375 (18.7) & $1553(19.9)$ & $4788(13.9)$ & $1665(14.5)$ \\
\hline Gestational $\mathrm{DM}^{\dagger}$ & $5458(23.3)$ & $1781(22.8)$ & $6639(19.3)$ & $2214(19.3)$ \\
\hline Chronic renal disease & $986(4.2)$ & $319(4.1)$ & $1402(4.1)$ & $514(4.5)$ \\
\hline Tobacco use & $1827(7.8)$ & $632(8.1)$ & $2992(8.7)$ & $1007(8.8)$ \\
\hline \multicolumn{5}{|l|}{$\begin{array}{l}\text { Medication } \\
\text { exposure }\end{array}$} \\
\hline Insulin & $2672(11.4)$ & $986(12.6)$ & $2843(8.3)$ & $1001(8.7)$ \\
\hline Oral diabetes medications & $3698(15.8)$ & $1398(17.9)$ & $2636(7.7)$ & $1006(8.8)$ \\
\hline
\end{tabular}

Am J Obstet Gynecol. Author manuscript; available in PMC 2016 March 01. 


\section{Table 3}

The association between chronic hypertension (treated and untreated) and congenital malformations compared with normotensive controls. Odds ratios and $95 \%$ confidence intervals shown.

\begin{tabular}{|c|c|c|c|c|}
\hline & \multicolumn{2}{|c|}{ Treated chronic hypertensives } & \multicolumn{2}{|c|}{ Untreated chronic hypertensives } \\
\hline & Unadjusted & PS-matched & Unadjusted & PS-matched \\
\hline $\begin{array}{l}\text { Composite congenital malformations } \\
\text { Organ-specific malformation }\end{array}$ & $1.7(1.6$ to 1.9$)$ & $1.3(1.2$ to 1.5$)$ & $1.5(1.4$ to 1.6$)$ & $1.2(1.1$ to 1.3$)$ \\
\hline Central nervous system malformations & $2.0(1.3$ to 3$)$ & $1.4(0.8$ to 2.3$)$ & $1.4(0.9$ to 2.1$)$ & $1.2(0.7$ to 1.9$)$ \\
\hline Malformations of the eye, ear, neck, or face & $0.9(0.5$ to 1.7$)$ & $0.8(0.4$ to 1.8$)$ & $1.1(0.7$ to 1.9$)$ & $1.2(0.7$ to 2.1$)$ \\
\hline Cardiac malformations & $2.6(2.3$ to 3$)$ & $1.6(1.4$ to 1.9$)$ & $2.1(1.9$ to 2.3$)$ & $1.5(1.3$ to 1.7$)$ \\
\hline Respiratory malformations & $1.8(1.2$ to 2.7$)$ & 1.5 (0.9 to 2.4$)$ & $1.4(0.9$ to 2$)$ & $1.3(0.8$ to 2.1$)$ \\
\hline Cleft palate and lip & $1.3(0.7$ to 2.3$)$ & $1.3(0.6$ to 2.6$)$ & $1.1(0.7$ to 1.9$)$ & $1.1(0.6$ to 2.1$)$ \\
\hline Gastrointestinal malformations & $1.1(0.8$ to 1.5$)$ & $1.0(0.7$ to 1.5$)$ & $1.1(0.9$ to 1.4$)$ & $1.0(0.7$ to 1.3$)$ \\
\hline Genitourinary malformations & $1.4(1.1$ to 1.8$)$ & $1.1(0.8$ to 1.5$)$ & $1.3(1.1$ to 1.7$)$ & $1.1(0.9$ to 1.5$)$ \\
\hline Musculoskeletal malformations & $1.1(0.9$ to 1.4$)$ & $0.9(0.7$ to 1.2$)$ & $1.0(0.8$ to 1.2$)$ & $0.8(0.7$ to 1.1$)$ \\
\hline Other malformations & $1.8(1.3$ to 2.4$)$ & $1.6(1.1$ to 2.3$)$ & $1.1(0.8$ to 1.5$)$ & $1.0(0.7$ to 1.4$)$ \\
\hline
\end{tabular}




\section{Table 4}

Sensitivity and exploratory analyses examining the association between chronic hypertension (treated and untreated) and congenital malformations compared with normotensive controls in the propensity-score matched cohorts. Odds ratios and $95 \%$ confidence intervals shown.

\begin{tabular}{|c|c|c|}
\hline & $\begin{array}{c}\text { Treated chronic } \\
\text { hypertensives } \\
\text { PS-matched }\end{array}$ & $\begin{array}{c}\text { Untreated chronic } \\
\text { hypertensives } \\
\text { PS-matched }\end{array}$ \\
\hline \multicolumn{3}{|c|}{$\begin{array}{l}\text { Sensitivity analysis 1--excluding } \mathrm{DM}^{*}, \mathrm{GDM}^{\dagger} \text {, insulin, } \\
\text { diabetes medication }\end{array}$} \\
\hline Composite congenital malformations & $1.2(1$ to 1.4$)$ & $1.2(1.1$ to 1.3$)$ \\
\hline Cardiac malformations & $1.5(1.2$ to 1.8$)$ & $1.5(1.2$ to 1.7$)$ \\
\hline \multicolumn{3}{|c|}{ Sensitivity analysis 2--exclude preterm deliveries } \\
\hline Composite congenital malformations & $1.3(1.1$ to 1.6$)$ & $1.1(1$ to 1.3$)$ \\
\hline Cardiac malformations & $1.5(1.2$ to 1.9$)$ & $1.1(0.9$ to 1.4$)$ \\
\hline \multicolumn{3}{|c|}{$\begin{array}{l}\text { Sensitivity analysis } 3 \text {-all covariates defined based on } \\
\text { trimester } 1\end{array}$} \\
\hline Composite congenital malformations & $1.3(1.2$ to 1.6$)$ & $1.2(0.9$ to 1.6$)$ \\
\hline Cardiac malformations & $1.6(1.3$ to 1.9$)$ & $1.3(0.9$ to 1.9$)$ \\
\hline \multicolumn{3}{|c|}{$\begin{array}{l}\text { Sensitivity analysis 4-outcome defined by a single } \\
\text { diagnostic code in the infant record }\end{array}$} \\
\hline Composite congenital malformations & $1.2(1.1$ to 1.3$)$ & $1.2(1.1$ to 1.3$)$ \\
\hline Cardiac malformations & $1.4(1.3$ to 1.6$)$ & $1.5(1.3$ to 1.6$)$ \\
\hline \multicolumn{3}{|c|}{ Exploratory analysis of specific cardiac malformations } \\
\hline Ventricular septal defect & $1.4(1.0$ to 2.0$)$ & $1.1(0.8$ to 1.5$)$ \\
\hline Right ventricular outflow obstruction & $1.2(0.2$ to 6.2$)$ & $1.3(0.4$ to 3.5$)$ \\
\hline Single ventricle & $3.0(0.4$ to 21.3$)$ & $4.5(0.8$ to 26.9$)$ \\
\hline Secundum atrial septal defect & $1.5(1.0$ to 2.4$)$ & $1.3(0.9$ to 1.9$)$ \\
\hline Conotruncal defect & $1.7(0.8$ to 3.5$)$ & $0.9(0.4$ to 1.8$)$ \\
\hline Left ventricular outflow obstruction & $1.8(0.8$ to 4.1$)$ & $1.1(0.5$ to 2.2$)$ \\
\hline
\end{tabular}

